\title{
SUR UN DÉVELOPPEMENT EN FRACTION CONTINUE \\ PAR
}

CH. HERMITE et L. FUCHS.

1. Extrait d'une lettre adressée à M. Hermite par M. Fuchs.

Peut-ètre vous intéressera-t-il de voir la manière dont je me suis démontré votre théorème ainsi énoncé:

DSoit $\alpha$ et $\beta$ deux expósants dont la somme $\alpha+\beta=k, k$ étant entier et positif, et $\frac{B}{A}$ la réduite d'ordre $n$ du développement en fraction continue de $(x-a)^{\alpha}(x-b)^{\beta}$. Les polynômes $A$ et $B$, des degrés $n$ et $n+k$, se déterminent sauf un facteur constant en posant:

$$
\begin{gathered}
D_{x}^{n}\left[(x-a)^{n+a}(x-b)^{n+\hat{\beta}}\right]=(x-a)^{\alpha}(x-b)^{\hat{\beta}} A \\
D_{x}^{n+k}\left[(x-a)^{n+k-a}(x-b)^{n+k-\beta}\right]=(x-a)^{-\alpha}(x-b)^{-\hat{\beta}} B .
\end{gathered}
$$

D'abord comme on peut changer l'expression $(x-a)^{a}(x-b)^{3}$ au moyen d'une substitution linéaire et entière en $t^{\alpha}(1-t)^{3}$, je considère immédiatement une telle expression, ou plutôt $x^{\lambda}(\mathrm{I}-x)^{\prime \prime}$, en mettant $\lambda$ et $\mu$ au lieu de $\alpha$ et $\beta$.

.Je me restreindrai à la démonstration de la formule (I), parce qu'on peut procéder de la mêtne inanière pour prouver la seconde. 
Il suit d'une formule donnće par $\mathrm{J}_{\Lambda \mathrm{COBI}}$, dans un mémoire posthume (.Journal de Borchariot 'T. 56, p. 149, § 3), que l'on a l'équation:

$$
D_{n}^{n}\left[x^{n+2}(1-x)^{n+n}\right]
$$

$=(\mathrm{I}+\lambda)(2+\lambda) \ldots(n+\lambda)^{\prime} x^{2}(\mathrm{I}-x)^{n} F(-n, k+n+\mathfrak{I}, \mathbf{I}+\lambda, x)$.

Or on peut poser:

(2) $x^{\prime}(\mathrm{I}-x)^{n}=G_{k}(x)+\frac{k(k-1) \ldots(k-k+\mathrm{I})}{1.2 \ldots k} F\left(\lambda, \mathrm{I}, \mathrm{I}+k, \frac{\mathrm{I}}{x}\right)$,

$G_{k}(x)$ étant un polynôme entier dı degré $k$. En dévoloppant

$$
F\left(\lambda, \mathrm{I}, k+1, \frac{1}{x}\right)
$$

en fraction continue, on toit que le dénominateur de la réduite d'ordre $n$ est identique à la quantité $A$ (sauf un facteur constant), et l'on déduit des formules de Heine (Journal de BonchardT T. 57, p. 231, et aussi Handbuch der Kugelfunctionen, T. I, chap. V), sauf un facteur constant:

$$
A=F(-n, k+n+\mathrm{I}, \mathrm{I}+\lambda, x) \text {. }
$$

On peut donc substituer dans l'équation ( 1 ) à la fonction

$$
F(-n, k+n+\mathrm{I}, \mathbf{I}+\lambda, x)
$$

la quantité $A$, ce qui démontre la premiere de ros deux formules.

Heidelberg, I 7 Octobre 1883 . 
2. Extrait d'une lettre adressée à M. Fuchs par M. Hermite.

..... Je me permets maintenant de vous communiquer une autre manière de parvenir au résultat que vous avez établi. Sous ce nouveau point de vue, je puis supposer $k$ indifférement positif ou négatif, j’admettrai seulement lorsque le second cas se presente que $n+k$ soit positif. Cela étant, je pose, en développant suivant les puissances déscendantes de la variable:

$$
(x-a)^{n+\alpha}(x-b)^{n+\hat{\beta}}=P+\frac{\varepsilon}{x}+\frac{\varepsilon^{\prime}}{x^{2}}+\ldots
$$

$P$ désignant la partie entière, et je prends les dérivées d'ordre $n$ des deux membres de cette égalité.

J'obtiens ainsi

$$
\begin{gathered}
D_{x}^{n}\left[(x-a)^{n+\alpha}(x-b)^{n+\hat{i}}\right]=(x-a)^{\mu}(x-b)^{\dot{s} A} \\
=I_{x}^{n} P+\frac{\eta}{x^{n+1}}+\frac{\eta^{\prime}}{x^{n+2}}+\ldots
\end{gathered}
$$

et cette relation met immédiatement en évidence que $\frac{D_{x}^{n} p}{A}$ est la réduite d'ordre $n$ du développement en fraction continue de la quantité

$$
(x-a)^{a}(x-b)^{\prime}
$$

le numérateur étant du degré. $n+k$, et le dénominateur du degré $n$.

On parvient à la même réduite, si l'on part de l'égalité suivante:

$$
(x-a)^{n+k-\alpha}(x-b)^{n+k-i x}=Q+\frac{\zeta}{x}+\frac{\xi^{\prime}}{x^{2}}+\ldots
$$

où la partie entière $Q$ est du degré $2 n+k$.

En prenant en effet la dérivéc d'ordre $n+k$ des deux membres, nous trouvons:

$$
(x-a)^{-a}(x-b)^{-\hat{\jmath}} B=D_{x}^{n+k} Q+\frac{y}{x^{n+k+1}}+\frac{y^{\prime}}{x^{n+k+2}}+\ldots
$$


et comme tout i l'heure on en conclut que $\frac{D^{n+k} Q}{B}$ cot une réduite du développement de $(x-a)^{-u}(x-b)^{-i x}$.

La fraction inverse $\frac{B}{D_{r}^{n+k} Q}$ est par conséquent, d'après le degré de son dénominateur la réduite d'ordre $n$ de la quantité $(x-a)^{\prime \prime}(x-l)^{3}$, et vous voyez que vous pouve écrire, sauf un facteur constant:

$$
B=D_{\imath c}^{\prime \prime} P, \quad I_{x}^{n+k} Q=A .
$$

Ces relations mettent en évidence une liaison bien singulière et que jusqu'ici je n'ai point cherché à approfondir entre $P, Q, A$ et $B$; je me contenterai d'avoir établi par la première le résultat que j’avais en vue.

Paris, I Février I 884 . 